\title{
Comparison of the short-term efficacy of sequential treatment with intravesical single-port laparoscopic partial cystectomy with bladder preservation or open partial cystectomy in combination with cisplatin plus gemcitabine chemotherapy
}

\author{
HAI-XING MAI ${ }^{1,2^{*}}$, JUN-LE LIU $^{2 *}$, SHU-JUN PEI $^{2 *}$, LI ZHAO $^{1}$, NAN QU $^{1}$, JIN-KAI DONG $^{1}$, \\ BIAO CHEN $^{1}$, YA-LIN WANG ${ }^{1}$, CHENG HUANG ${ }^{1}$ and LI-JUN CHEN ${ }^{2}$ \\ ${ }^{1}$ Chinese People's Liberation Army General Hospital \& Medical School of Chinese People's Liberation Army, \\ Beijing 100853; ${ }^{2}$ Department of Urology, Affiliated Hospital, Academy of Military Medical Sciences, \\ Beijing 100071, P.R. China
}

Received June 25, 2014; Accepted March 18, 2015

DOI: $10.3892 / \mathrm{etm} .2015 .2448$

\begin{abstract}
This study aimed to assess the short-term efficacy of sequential therapy for T2/T3a bladder cancer with intravesical single-port laparoscopic partial cystectomy or open partial cystectomy combined with cisplatin plus gemcitabine (GC) chemotherapy in a prospective randomized controlled study. Thirty patients with bladder cancer who underwent open partial cystectomy (group A) or single-port laparoscopic partial cystectomy (group B) and received standard GC chemotherapy were analyzed. Perioperative functional indicators and tumor recurrence during a 1-year postoperative follow-up were compared between the two groups. The baseline characteristics were comparable between the two groups. The mean operative time, amount of blood loss and duration of hospital stay were $90.3 \mathrm{~min}, 182.0 \mathrm{ml}$ and 7.3 days, respectively, for group A, and $105.3 \mathrm{~min}, 49.3 \mathrm{ml}$ and 5.8 days, respectively, for group B. No secondary postoperative bleeding, urine leakage, wound infection or other complications were observed in the two groups. Postoperative scarring was not evident in group B. The overall incidence of surgical complications, tumor recurrence rate and complications during chemotherapy in the postoperative follow-up period of 12 months were similar between the two groups. Single-port laparoscopic partial cystectomy surgery is an idea surgical method for the treatment of invasive bladder cancer, with good surgical effect, minimal invasiveness,
\end{abstract}

Correspondence to: Professor Li-Jun Chen, Department of Urology, Affiliated Hospital, Academy of Military Medical Sciences, 8 Dong Fengtai, Beijing 100071, P.R. China

E-mail: cnlijunchen@163.com

*Contributed equally

Key words: cystectomy, laparoscopy, single-port laparoscopy, bladder cancer rapid recovery and short hospital stay. The data from 1-year postoperative follow-up showed that laparoscopic surgery was superior with regard to perioperative bleeding, postoperative recovery and duration of indwelling urinary catheter use. However, regarding the tumor recurrence rate, long-term comparative details are required to determine the effect of laparoscopic surgery.

\section{Introduction}

As a surgical method to preserve the bladder, partial cystectomy can be used to achieve full-thickness resection of the bladder wall and complete resection of bladder lesions, in addition to ensuring adequate margins. In the 1950s, partial cystectomy became widely used to treat muscle-invasive bladder cancer (MIBC); however, due to its high recurrence rate $(40-78 \%)$, together with the development of controllable urinary diversion technology and the application of total cystectomy with preservation of nerves and prostate, radical cystectomy has become the 'gold standard' for the treatment of MIBC (1-3). However, study results from a 30-year period indicate that the postoperative 5-year survival rate of radical cystectomy is only $40-60 \%$ (4). In addition, due to the high perioperative mortality rate $(1.5-4.2 \%)$ and incidence of complications $(67 \%)(5,6)$, which seriously affect the quality of life following urinary diversion and damage the sexual function of male patients after surgery, the 'gold standard' status for radical cystectomy has been challenged.

A study (7) has shown that $50 \%$ of MIBC has dominant or occult local or distant metastasis at the first diagnosis. For these patients whose prognoses cannot be significantly improved by modified surgical methods alone, combined treatment with radiotherapy and chemotherapy is a useful complement to surgery. Therefore, treatment with bladder preservation is likely to achieve similar long-term efficacy to that of radical cystectomy and a higher quality of life (8).

In 2008, Mak et al (9) indicated that the most common treatment methods with bladder preservation included 'thorough' 
transurethral resection of bladder tumor (TURBt), systemic chemotherapy (adjuvant or neoadjuvant chemotherapy) and radiotherapy. However, traditional TURBt has a blind zone and other deficiencies, such as the incapability to access tumors in particular areas, and is a challenging technique for the surgeon to master. Therefore, the clearance of localized tumors in MIBC is $<20 \%$ for TURBt alone, and phase T3 tumors are rarely resected completely by TURBt (10). Residual tumor and pathological downstaging following TURBt is not uncommon (11). For patients with invasive bladder cancer, TURBT alone can at most only reduce tumor burden, and alleviate symptoms such as frequent urination, urgency with urination and bloody urine; and $~ 50 \%$ of patients have distant metastases postoperatively (6).

By contrast, partial cystectomy enables complete resection of the full thickness of the lesion and ensures adequate safety margins, with a low incidence of residual tumor. In addition, the surgical procedure of partial cystectomy is relatively simple. However, the traditional open partial cystectomy surgery has several deficiencies such as high surgical risk, large trauma, long postoperative recovery and an evident postoperative scar. Laparoscopic surgery techniques have been developed since the early 1990s, offering the possibility for the reduction of complications in open surgery; with the unremitting effort of clinicians, laparoscopic surgery has been developed from the classic four-incision surgery (12) to three-incision surgery (13) and two-incision surgery (14), and ultimately single-port surgery has successfully been achieved.

For single-port laparoscopic surgery, natural orifice transumbilical surgery (NOTUS) is the most mature surgical method. The incision of NOTUS is located in the umbilical region where the incision is covered by the skin fold; therefore, satisfactory cosmetic results can be achieved. Furthermore, NOTUS has other advantages, including an absence of scarring, a superior cosmetic effect, less postoperative pain, rapid recovery and a lower incidence of trocar site hernia and infection. At present, the range of single-port laparoscopic surgery encompasses kidney cryotherapy, kidney biopsy, radical nephrectomy, single-port transumbilical nephrectomy for living kidney transplantation donors, sleeve gastrectomy, gastric banding surgery, right hemicolectomy and other difficult surgeries (15-19).

Therefore, based on familiarity with single-port laparoscopic renal cyst decompression and adrenalectomy, the present authors attempted to apply the single-port laparoscopic technique to partial cystectomy and the results obtained are reported in the present study.

\section{Materials and methods}

Patient data. The study population included 30 patients who underwent surgical treatment due to bladder cancer while admitted to the Affiliated Hospital of the Academy of Military Medical Sciences (Beijing, China) between June 2011 and July 2013 were included in this study. The selected patients were diagnosed with bladder cancer with total cystectomy indications. The patients themselves and their families had expressly rejected total cystectomy.

The 30 patients included 18 males and 12 female patients, with a mean age of 63.6 years (range, $45-81$ years). The tumor stages were T2 in 11 cases and T3a in 19 cases. All patients were confirmed by preoperative cystoscopy biopsy as having high-grade invasive bladder cancer, with pathological grades of II (16 cases) or III (14 cases; Fig. 1). The number of tumor lesions was 1-4, with a maximum tumor diameter of $4.5 \mathrm{~cm}$. Systemic metastases were excluded by routine chest radiograph, abdominal B-ultrasound, pelvic computed tomography (CT) scan and whole body bone emission CT scan (Fig. 2). The patients all underwent sequential therapy with bladder preservation.

The included patients were randomly divided into two groups, and were randomized by random numbers assigned by computer. This study was conducted in accordance with the Declaration of Helsinki. This study was conducted with approval from the Ethics Committee of the Academy of Military Medical Sciences (Beijing, China). Written informed consent was obtained from all participants.

Single-port laparoscopic partial cystectomy. The surgical instruments used included TriPort (Olympus Corporation, Tokyo, Japan), an ultrasonic scalpel, $10-\mathrm{mm} 30^{\circ}$ laparoscope, 2-mm spring grasping forceps, needle holders, curved electrocautery hook, 5-mm straight scissors and 5-mm washer (Karl Storz GmbH \& Co. KG, Tuttlingen Germany). The patient was laid in a supine lithotomy position with the head at $30^{\circ}$, and the location and size of the tumor was determined by cystoscopy, when the operator stood at the patient's cephalic position and the assistant stood at the contralateral position to the operator to hold the cystoscope. When placing the single-port multi-channel kit, a longitudinal skin incision was made $2 \mathrm{~cm}$ below the belly button, and the skin fold was incised. The peritoneum was pushed upward to expose the top of bladder. Under the surveillance of the cystoscope and with maintenance of bladder filling, the top wall of bladder was punctured and the single-port multi-channel kit was put in place following positioning by fine needle puncture. The cystoscope was then withdrawn, and an independent intake passage was connected to a pneumoperitoneum apparatus (KARL STORZ Endoscopy [Shanghai] Ltd., Beijing, China) to establish a gas-filled bladder space with a pressure of $12-15 \mathrm{mmHg}$. The locations and sizes of tumors in the bladder were observed after filling the bladder, and the tumors were incised completely at about a distance of $1-2 \mathrm{~cm}$ from the tumor basal margin up to the muscular layer of the bladder using the electrocautery hook and ultrasonic scalpel. The bladder incision was sutured using 2.0 absorbable suture lines if necessary, and the surgical specimens were placed in specimen bags. Following the surgery, the surgical wound and incision were soaked and rinsed thoroughly with distilled water, and the bladder and abdominal incision were closed layer by layer. An indwelling urinary catheter was used for continuous bladder irrigation.

Open partial cystectomy. The patient was laid in a supine position, and a central incision was made below the umbilicus with a length of $\sim 15 \mathrm{~cm}$. The skin, subcutaneous tissue and muscle layer were incised layer by layer, and the peritoneum was bluntly dissected to expose the bladder. The tissue surrounding the bladder was separated by a combination of blunt and sharp dissection combined and the bladder was incised. The tumors in the bladder were located and completely resected at a 
Table I. Patient characteristics (mean \pm standard deviation, $\mathrm{n}=15$ per group).

\begin{tabular}{lccc}
\hline Parameters & Open surgery & Laparoscopy & P-value \\
\hline Age (years) & $64.600 \pm 8.943$ & $62.533 \pm 10.501$ & 0.5663 \\
Number of tumors (n) & $2.133 \pm 0.990$ & $2.200 \pm 0.862$ & 0.8455 \\
Volume of tumors (cm) & $2.667 \pm 0.900$ & $2.633 \pm 0.743$ & 2.8 \\
Follow-up duration (months) & $9.600 \pm 2.586$ & $9.000 \pm 2.268$ & 0.5048
\end{tabular}
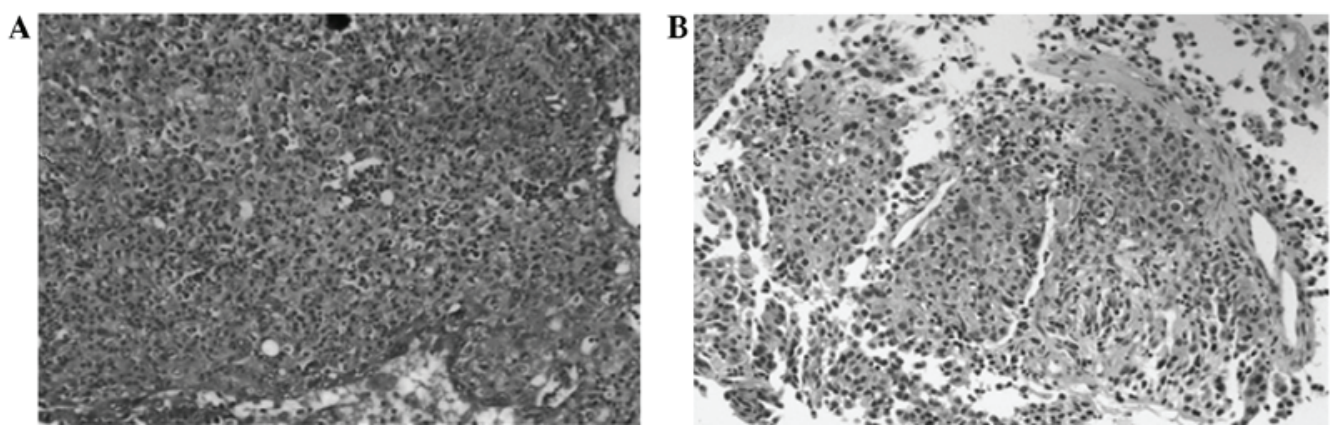

Figure 1. Invasive bladder cancer confirmed by biopsy. Grades (A) III and (B) II bladder cancer (magnification, x200)
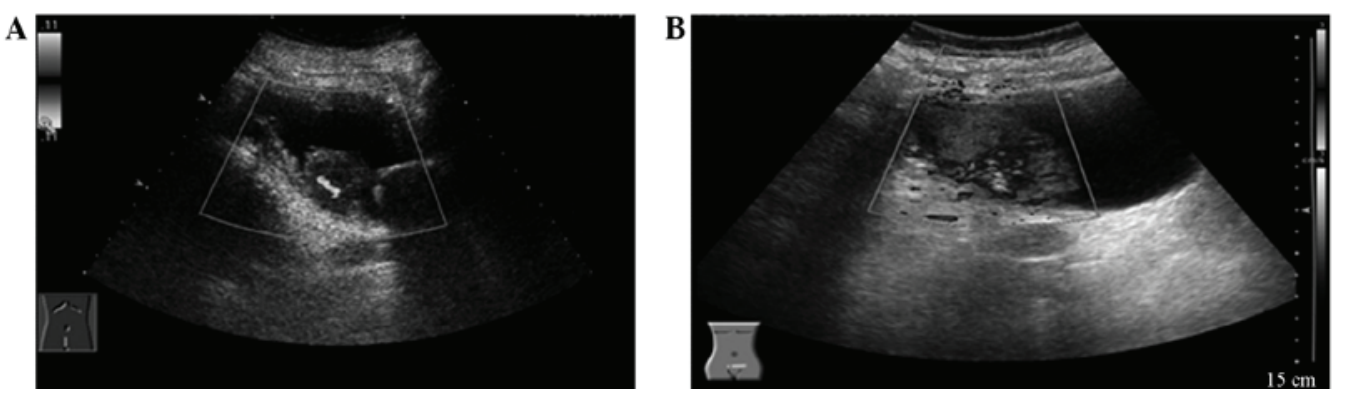

Figure 2. Ultrasound image displaying (A) the tumor size and number, and (B) the blood supply of the bladder tumor.

distance of $\sim 1 \mathrm{~cm}$ around the tumor. The excised specimens were sent for pathological examination. The surgical wound was rinsed with distilled water and saline, and an indwelling urinary catheter was inserted. The bladder incision was continuously sutured using 2.0 absorbable suture lines and embedded using No. 1 silk after confirming that there was no active hemorrhage in the bladder. An indwelling silicone drainage tube was inserted beside the incision following the confirmation that there was no urine leakage or hemorrhage, and the incision was closed layer by layer.

Chemotherapy regimen. The systemic chemotherapy regimen (cisplatin plus gemcitabine hydrochloride; GC regimen) was gemcitabine hydrochloride $1 \mathrm{~g} / \mathrm{m}^{2}$ (days 1,8 and 15) and cisplatin $70 \mathrm{mg} / \mathrm{m}^{2}$ (day 2), with 3 weeks for 1 treatment course. Cystoscopy, pelvic B ultrasound and pelvic CT were performed at follow-up intervals (once every 3 months) to check for tumor recurrence.

Statistical analysis. The results were recorded in EXCEL spreadsheets (Microsoft, Seattle, WA USA) and analyzed using the SPSS software, version 16.0 (SPSS Inc., Chicago, IL, USA).
All result parameters are expressed as mean \pm standard deviation. A t-test was used to analyze the difference level between the two groups. Perioperative categorical data or complication results were analyzed by non-parametric Whitney $U$ test, $\chi^{2}$ test and Fisher's exact test. $\mathrm{P}<0.05$ was considered to indicate a statistically significant difference.

\section{Results}

Patient characteristics. The 30 patients were randomized to an open surgery group (group A) and laparoscopic surgery group (group B), with 15 patients in each group. All 30 patients were included in the statistical analysis. The baseline characteristics of the two groups included gender, mean age, clinical stage, pathological stage, number of tumors and maximum tumor diameter. The baseline characteristics were comparable between the two groups, and no difference with statistical significance was observed (Table I).

Perioperative situation and follow-up. The recurrence rate for the two groups during follow-up was 3/15 and 3/15 for groups A and B, respectively (Table II). Although there was no 
Table II. Follow-up data for the two groups (n=15 per group).

\begin{tabular}{lccr}
\hline Variable & Open surgery, $\mathrm{n}(\%)$ & Laparoscopic surgery, n $(\%)$ & P-value \\
\hline Tumor recurrence & $3 / 15(20)$ & $3 / 15(20)$ & 1.0000 \\
Frequent urination & $4 / 15(26.7)$ & $2 / 15(13.3)$ & 0.3613 \\
Incision infection & $3 / 15(20)$ & $0 / 15(0)$ & 0.0679 \\
Grade I hematological toxicity & $8 / 15(53.3)$ & $7 / 15(46.7)$ & 0.715 \\
Grade II hematological toxicity & $3 / 15(20)$ & $4 / 15(26.7)$ & 0.0614 \\
Grade III hematological toxicity & $2 / 15(13.3)$ & $3 / 15(20)$ & 0.0162 \\
Grade IV hematological toxicity & $2 / 15(13.3)$ & $1 / 15(6.7)$ & 0.0060 \\
\hline
\end{tabular}

Table III. Perioperative data (mean \pm standard deviation, $\mathrm{n}=15$ per group).

\begin{tabular}{lccc}
\hline Variable & Open surgery & Laparoscopic surgery & P-value \\
\hline Duration of surgery (min) & $90.333 \pm 16.740$ & $105.333 \pm 11.255$ & 0.0075 \\
Surgical blood loss (ml) & $182.000 \pm 59.546$ & $49.333 \pm 14.864$ & $<0.0001$ \\
Duration of drainage (days) & $4.067 \pm 1.387$ & $1.20 \pm 0.941$ & 0.0002 \\
Duration of urinary catheterization (days) & $7.333 \pm 0.900$ & $3.800 \pm 0.775$ & 0.0000 \\
Hospital stay (days) & $7.267 \pm 1.033$ & $5.800 \pm 1.699$ & 0.0080 \\
\hline
\end{tabular}
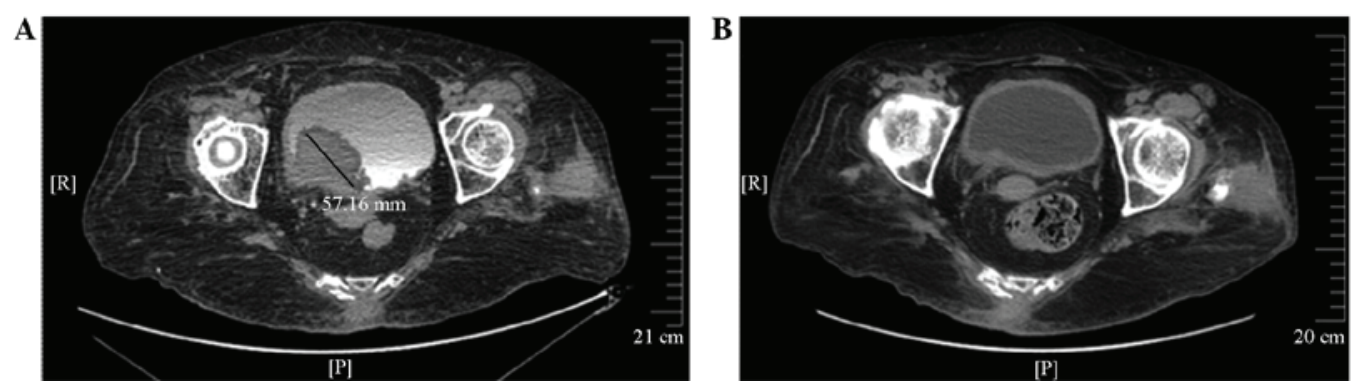

Figure 3. Computed tomography (CT) examination prior to and following treatment. (A) Pre-treatment CT image showing the size of bladder cancer is $\sim 57 \mathrm{~mm}$, with small tumors near the primary tumor. (B) CT image captured after 1 year of follow-up displaying that the tumor had moved up, and the bladder mucous membrane was smooth, with no tumor recurrence.

significant difference in overall survival rate and the incidence of surgical incision infection between the two groups, the degree of bleeding was notably higher in group A compared with group B (Table II and Fig. 3). The incidence of early postoperative frequent urination was notably higher in group A compared with group B (4 patients in group A and 2 patients in group B), but the incidence rate between the two groups showed no significant difference $(\mathrm{P}=0.36)$. The symptom of frequent urination in group A was mostly relieved in 1 month after surgery, although in 1 patient the symptom was relieved 3 months after surgery. According to the Clavien-Dindo classification (20) of surgical complications, no significant difference was observed in the category and level of complications between the two groups. All 30 patients successfully completed the scheduled chemotherapy regimen During follow-up, bladder function was sustained and no tumor recurrence was detected (Fig. 3). All the patients experienced a high quality of life. None of the patients had severe hematological toxic reactions during chemotherapy, and the complications were improved following symptomatic treatments. The hematological toxic reactions included: 8 cases of grade I; 3 cases of grade II, 2 cases of grade III; and 2 cases of grade IV in group A; and 7 cases of grade I, 4 cases of grade II, 3 cases of grade III and 1 case of grade IV in group B (Table II).

Follow-up data and postoperative complications.Perioperative data are summarized in Table III. The duration of surgery was significantly longer in group B than in group A; however, the amount of surgical blood loss, the duration of postoperative urinary catheter and drainage pipe indwelling and hospital stay were significantly greater in group A than in group B.

\section{Discussion}

Over the past decade, as the understanding of bladder cancers, particularly of muscle-invasive bladder cancers, has deepened, it has been realized that bladder cancer is a systemic disease that cannot be cured by local surgical treatment alone (7). 
Combination treatment with radiotherapy and chemotherapy is an effective method of improving the long-term survival of invasive bladder cancer following surgery (8).

Zhang et al (21) performed a retrospective analysis of the treatment and follow-up results of 136 cases of MIBC in Shanghai Ruijin Hospital between 2002 and 2007. It was observed that the 5-year cancer-specific survival rate was higher in the patients that underwent partial cystectomy than in those who underwent radical cystectomy (68 vs. 55\%, respectively; $\mathrm{P}=0.033$ ), and the 5-year cancer-specific survival rate in all MIBC patients was $65 \%$. The mean cancer-specific survival times in all MIBC cases and the partial and radical cystectomy groups were 49.9, 52.6 and 40.4 months, respectively; 75 and $90 \%$ of local recurrence occurred between 16 and 32 months after surgery, respectively.

In 2004, Holzbeierlein et al (22) summarized the results of 58 patients with MIBC treated with partial cystectomy in the Memorial Sloan-Kettering Cancer Center from 1995 to 2001, and observed that the progressive recurrence-associated risk factors included concomitant carcinoma in situ (CIS) [odds ratio $(O R)=7.05$ ] and positive regional nodes $(\mathrm{OR}=4.38)$. In 2006, Kassouf et al (23) reported that pathological staging was associated with recurrence, and the history of superficial bladder cancer was associated with clinical staging and progressive recurrence.

In 2008, Smadlone et al (24) reported the results of 25 patients with MIBC who received partial cystectomy from 1995 to 2005. The mean follow-up duration was 45.3 months (8-118 months), and the 5-year cancer-specific survival rate was $84 \%$. During the follow-up period, the recurrence rate was $28 \%$, of which $8 \%$ was superficial recurrence and $20 \%$ was progress recurrence. The tumor volume was the only risk factor that was found to be associated with recurrence. The treatment of MIBC with partial cystectomy remains a controversial topic, with the focus of controversy lying in the appropriate indications. The indications for the treatment of MIBC with partial cystectomy recommended in the 2011 edition of the US NCCN bladder cancer treatment guidelines are: Single T2 tumor with appropriate location and not associated with CIS. The proportion of clinical cases of MIBC meeting this stringent standard is $<5 \%$ (21). However, this surgical indication can be relaxed if sequential treatment with partial cystectomy combined with the GC regimen is used. In the present study, the recurrence rate was not significantly different between the open surgery group and the laparoscopic surgery group based on 1-year follow-up data (3/15 vs. $3 / 15$, respectively). The majority of the recurrence in these patients was local recurrence, which is consistent with the results of Zhang et al (21). The primary risk factors for recurrence of bladder cancer are the primary tumor size and tumor pathological grade.

With the application of laparoscopy in urology and the improvement of intraluminal suture techniques, laparoscopic techniques have been applied in cystectomy or partial cystectomy. It has been confirmed in clinical practice that these two types of procedure are technically feasible, with several advantages such as reduced trauma, less blood loss, mild postoperative pain, shorter hospital stay, better surgical field appearance and rapid recovery with the resumption of normal daily activities (25). Conventional partial cystectomy surgery requires the use of 3-5 channels, which requires numerous skin incisions, provides poor cosmetic results and has a relatively high risk of peritoneal implantation. To further reduce trauma, improve cosmetic results and reduce tumor metastasis during the surgical procedure, a single-port laparoscopic technique has been proposed. Single-port multi-channel operating systems and special instruments, such as flexible endoscopy, grasping forceps, scissors and needle holders, have been successfully developed and have laid the foundation for single-hole multi-channel laparoscopic techniques $(16,26)$.

In 2006, Chen et al (27) reported 8 cases of bladder cancer that were successfully treated by intraperitoneal laparoscopic partial cystectomy, with a mean surgery time of $230 \mathrm{~min}$, mean blood loss volume of $300 \mathrm{ml}$ and mean hospital stay of 7 days. No intraoperative and postoperative complications occurred. Two patients had local recurrence and distant metastasis 12 and 14 months after surgery, respectively. Conservative treatment was provided. The other 6 cases had no tumor recurrence and distant metastasis during the 8-22 month follow-up period.

Based on the mastery of single-port laparoscopy for renal cyst decortication and adrenalectomy, single-port laparoscopic partial cystectomy has been conducted in the Affiliated Hospital of the Academy of Military Medical Sciences. The postoperative results have been satisfactory, with a duration of surgery of 90-120 min (mean, $105.3 \mathrm{~min}$ ), intraoperative bleeding volume of 20-70 $\mathrm{ml}$ (mean, $49.3 \mathrm{ml}$ ), and mean hospital stay of 5.8 days. The wound drainage tube was removed on the day following the surgery, and the urethral catheter was removed within 5 days after surgery.

In the surgical procedure, it was recognized that single-port laparoscopic partial cystectomy has unique advantages for the treatment of bladder cancer. Firstly, the bladder was filled with gas via the top of the bladder using a pneumoperitoneum apparatus, which avoided interference with the abdominal organs and reduced intestinal adhesion and wound complications. The gas-filled bladder acted as a retractor, which facilitated the manipulation of the instruments and resulted in improved surgical efficiency. In addition, the gas-filled bladder had a beneficial role in hemostasis, by compression of the bladder wall to reduce the bleeding that is the main problem of open TURBt. Secondly, the majority of the laparoscopic surgery procedure was conducted under direct vision, with which the blind zone of traditional open surgery and TURBt could be effectively observed and treated. By aspirating urine and intraoperative bleeding through a urinary catheter in the bladder and using barbed absorbable suture techniques, the duration of surgery was reduced. Thirdly, the extension and depth of surgical resection were comparable to those of traditional open surgery, and it was possible to confirm the intraoperative bleeding site, conduct effective hemostasis, and conduct intraluminal suturing for resected sites. Following an initial attempt, it was also found that compared with conventional laparoscopy or other intraluminal surgeries such as transurethral resection of the prostate, the transvesical single-port laparoscopic partial cystectomy surgery was relatively challenging, and the learning curve was relatively long requiring special training and the use of certain flexible instruments to successfully complete the surgery. The duration of surgery was longer when larger tumors were resected, and when complete resection of the tumor tissue was expected, it was necessary to appropriately expand the incision or cut the tissue prior to removal of the tumor. 
With regard to the procedures used in the surgery, the following recommendations are made based on experience: i) The bladder puncture site should avoid the peritoneum to reduce the impact on the abdominal viscera; ii) the placement of the single-port kit should be conducted under the direct vision of the cystoscope, with maintenance of satisfactory bladder filling; iii) the use of a 5-mm integrated endoscope can reduce the interference of the surgery by the instruments; iv) the ureteral open position should be confirmed prior to the resection of the bladder tumor to avoid injury; v) mastery of the intraluminal suture technique is necessary; vi) ureteral reimplantation should be considered if the distance from the tumor base to the ureteral orifice is $<\mathrm{cm}$, or in cases of accidental ureter damage; vii) for large resection tissue blocks, a morcellator can be used or the tissue can be split into smaller pieces prior to the removal of the tissue.

The following subjects remain controversial in laparoscopic partial cystectomy: The risk of intra-abdominal tumor implantation; urine leakage and ureteral injury; and bladder tumor recurrence. However, these risks of surgery can be avoided by the use of single-port laparoscopy. The laparoscopic technique described in the present study used an extraperitoneal approach, which avoided the flowing of urine into the abdominal cavity. In addition, filling the bladder with water to observe the peritoneal position and fully separating the peritoneum is conducive to bladder suturing. Cystoscopy performed during the surgery to determine the location of the tumor and select the optimum puncture site prevents penetration of the tumor tissue and reduces puncture channel transfer. With monitoring by laparoscopy, the tumor can be completely resected using an electrocautery hook and ultrasonic scalpel; thus, the surgery may be conducted precisely and with minimal damage. Furthermore, under the pressure of the pneumoperitoneum, the bladder mucosa can be fully developed, so that during the surgical procedure it is possible to fully observe the bladder mucosa. For smaller tumors in the bladder and tumors missed by cystoscopic examination, electrocautery gasification can be conducted using an electrocautery hook, and for tumors on the side-wall of the bladder, a ureteral catheter can be inserted during surgery to prevent damage of the ureteral orifice. During surgery, 2.0 absorbable suture lines can be used, depending on the circumstances, for continuous full-thickness suturing of the bladder, followed by injection supercharging of the bladder to observe whether there is any leakage of urine. In order to reduce the implantation of tumor cells in the extraperitoneal space, the incised tumor specimens should be placed in a specimen bag as quickly as possible, and the extraperitoneal space should be soaked in distilled water for $10 \mathrm{~min}$ prior to suturing the bladder. The patients in this study had no peritoneal damage, the specimens were all complete, $>1.5 \mathrm{~cm}$ and the bladder healed well without any urine leakage or ureteral damage. The drainage tubes were removed 24-48 $\mathrm{h}$ after surgery.

In summary, transvesical single-port laparoscopic partial cystectomy has been found to be a safe, effective and feasible surgical method following its initial application in bladder surgery. This surgical method not only is advantageous compared with open surgery and transurethral surgery, but also compensates for the deficiencies of these two traditional surgical methods. The single-port laparoscopic method is not only minimally invasive (less invasive, with better cosmetic results than ordinary multiple-port laparoscopy), but also avoids the risk of water intoxication that traditional transurethral surgery has, in addition to the deficiencies of incompletely tumor resection and poorer long-term efficacy. Based on experience in China and overseas, it is considered that with regard to the 'gold standard' status of radical cystectomy for the treatment of MIBC, in appropriate cases of MIBC, surgery with bladder preservation is a practical and significant method for improving the quality of life and reducing the risk of surgery for patients. As a simple and safe surgical method, with few complications that enables the preservation of the bladder, single-port laparoscopic partial cystectomy has unique advantages, which are worthy of further exploration and may be recommended for wider use. Due to the current results being from a small sample at a single center, further controlled clinical studies with a long-term follow-up are required to confirm the clinical efficacy.

\section{References}

1. Lindahl F, Jørgensen D and Egvad K: Partial cystectomy for transitional cell carcinoma of the bladder. Scand J Urol Nephrol 18: 125-129, 1984.

2. Resnick MI and O'Conor VJ Jr: Segmental resection for carcinoma of the bladder: Review of 102 patients. J Urol 109: 1007-1010, 1973

3. Novick AC and Stewart BH: Partial cystectomy in the treatment of primary and secondary carcinoma of the bladder. J Urol 116: 570-574, 1976.

4. Oosterlinck W, Lobel B, Jakse G, Malmström PU, Stockle M and Sternberg C; European Association of Urology (EAU) Working Group on Oncological Urology: Guidelines on bladder cancer. Eur Urol 41: 105-112, 2002.

5. Shabsigh A, Korets R, Vora KC, et al: Defining early morbidity of radical cystectomy for patients with bladder cancer using a standardized reporting methodology. Eur Urol 55: 164-174, 2009.

6. Ramani VA, Maddineni SB, Grey BR and Clarke NW: Differential complication rates following radical cystectomy in the irradiated and nonirradiated pelvis. Eur Urol 57: 1058-1063, 2010.

7. Merseburger AS, Matuschek I and Kuczyk MA: Bladder preserving strategies for muscle-invasive bladder cancer. Curr Opin Urol 18: 513-518, 2008.

8. Solsona E, Climent MA, Iborra I, et al: Bladder preservation in selected patients with muscle-invasive bladder cancer by complete transurethral resection of the bladder plus systemic chemotherapy: Long-term follow-up of a phase 2 nonrandomized comparative trial with radical cystectomy. Eur Urol 55: 911-919, 2009.

9. Mak RH, Zietman AL, Heney NM, Kaufman DS and Shipley WU: Bladder preservation: Optimizing radiotherapy and integrated treatment strategies. BJU Int 102: 1345-1353, 2008.

10. Merseburger AS and Kuczyk MA: The value of bladder-conserving strategies in muscle-invasive bladder carcinoma compared with radical surgery. Curr Opin Urol 17: 358-362, 2007.

11. Divrik RT, Sahin AF, Yildirim U, Altok M and Zorlu F: Impact of routine second transurethral resection on the long-term outcome of patients with newly diagnosed pT1 urothelial carcinoma with respect to recurrence, progression rate and disease-specific survival: A prospective randomised clinical trial. Eur Urol 58: 185-190, 2010.

12. Bauer O, Devroey P, Wisanto A, Gerling W, Kaisi M and Diedrich K: Small diameter laparoscopy using a microlaparoscope. Hum Reprod 10: 1461-1464, 1995.

13. Leggett PL, Bissell CD, Churchman-Winn R and Ahn C: Three-port microlaparoscopic cholecystectomy in 159 patients. Surg Endosc 15: 293-296, 2001.

14. Kagaya T: Laparoscopic cholecystectomy via two ports, using the 'Twin-Port' system. J Hepatobiliary Pancreat Surg 8: 76-80, 2001.

15. Kaouk JH, Haber GP, Goel RK, et al: Single-port laparoscopic surgery in urology: Initial experience. Urology 71: 3-6, 2008. 
16. Kaouk JH and Palmer JS: Single-port laparoscopic surgery: initial experience in children for varicocelectomy. BJU Int 102: 97-99, 2008.

17. Reavis KM, Hinojosa MW, Smith BR and Nguyen NT: Single-laparoscopic incision transabdominal surgery sleeve gastrectomy. Obes Surg 18: 1492-1494, 2008.

18. Nguyen NT, Hinojosa MW, Smith BR and Reavis KM: Single laparoscopic incision transabdominal (SLIT) surgery-adjustable gastric banding: A novel minimally invasive surgical approach. Obes Surg 18: 1628-1631, 2008.

19. Remzi FH, Kirat HT, Kaouk JH and Geisler DP: Single-port laparoscopy in colorectal surgery. Colorectal Dis 10: 823-826, 2008.

20. Tokunaga M, Kondo J, Tanizawa Y, Bando E, Kawamura T and Terashima M: Postoperative intra-abdominal complications assessed by the Clavien-Dindo classification following open and laparoscopy-assisted distal gastrectomy for early gastric cancer. J Gastrointest Surg 16: 1854-1859, 2012.

21. Zhang M, Tao R, Zhang C and Shen Z: Lymphovascular invasion and the presence of more than three tumors are associated with poor outcomes of muscle-invasive bladder cancer after bladderconserving therapies. Urology 76: 902-907, 2010.
22. Holzbeierlein JM, Lopez-Corona E, Bochner BH, et al: Partial cystectomy: A contemporary review of the Memorial Sloan-Kettering Cancer Center experience and recommendations for patient selection. J Urol 172: 878-881, 2004.

23. Kassouf W, Swanson D, Kamat AM, et al: Partial cystectomy for muscle invasive urothelial carcinoma of the bladder: A contemporary review of the M. D. anderson cancer center experience. J Urol 175: 2058-2062, 2006

24. Smaldone MC, Jacobs BL, Smaldone AM and Hrebinko RL Jr: Long-term results of selective partial cystectomy for invasive urothelial bladder carcinoma. Urology 72: 613-616, 2008.

25. Chapron C, Dubuisson JB, Jacob S, Fauconnier A and Da Costa Vieira M: Laparoscopy and bladder endometriosis. Gynecol Obstet Fertil 28: 232-237, 2000 (In French).

26. Gill IS, Canes D, Aron M, et al: Single port transumbilical (E-NOTES) donor nephrectomy. J Urol 180: 637-641, 2008.

27. Chen X, Qi L, Chen HQ, Qi F, Liu HY and Chen XX: Laparoscopic partial cystectomy. Zhong Guo Nei Jing Za Zhi She 12: 456-457, 2006 (In Chinese). 